\title{
Comparaison de la croissance en hauteur entre 1 et 25 ans de 12 provenances de Douglas (Pseudotsuga menziesii (Mirb) Franco)
}

\author{
P Rozenberg \\ INRA Orléans, Station d'amélioration des arbres forestiers, 45160 Ardon, France
}

(Reçu le 18 mars 1992; accepté le 16 avril 1993)

\begin{abstract}
Résumé - On a comparé la croissance en hauteur de 12 provenances de Douglas entre 1 et 25 ans depuis la graine. La corrélation entre les classements sur la hauteur des provenances à 10 et à 25 ans dépend beaucoup de la composition du lot de provenances étudiées. Deux principales techniques de traitement des données ont été utilisées : méthodes de régression et analyses multifactorielles. La modélisation a permis de définir des groupes de provenances significativement différents pour la vitesse de croissance en hauteur. Les analyses multifactorielles ont mis en évidence que les provenances avaient des stratégies de croissance en hauteur différentes. Par exemple, la sécheresse de 1976 a ralenti la croissance de certaines d'entre elles. La connaissance des vitesses et stratégies de croissance en hauteur des provenances est utile pour choisir celles qui sont les mieux adaptées à des types de sylviculture donnés, notamment des couples densité-révolution. Les avantages respectifs de la modélisation et des analyses multifactorielles ont été discutés.
\end{abstract}

croissance / hauteur / provenance / Douglas / Pseudotsuga menziesil

Summary - Height growth of 12 Douglas fir (Pseudotsuga menziesil (Mirb) Parl) seed sources between 1 and 25 years old. Height growth of 12 Douglas fir seed sources has been compared using modelling (height-age curves) and multifactorial analysis. Modelling led to the definition of significantly different seed source groups based on height growth rate (table V). The multifactorial analysis showed that seed sources had different growth patterns (figs 6, 7), linked to genotypic and environmental effects. For example, the 1976 drought slowed down the growth of some but not all seed sources (figs 4, 5). Age-age correlations for height have been investigated (figs 2, 3). The seed sources growth speed and pattern are useful in choosing the seed sources best adapted to different sylviculture type, and in particular, couple spacing-rotation. Respective advantages of multifactorial analysis and modelling have been discussed.

growth height / seed source / Douglas fir / Pseudotsuga menziesii 


\section{INTRODUCTION}

L'étude de la variabilité naturelle du Douglas (Pseudotsuga menziesii (Mirb) Franco) a débuté en France en 1953 par l'installation de dispositifs de comparaison de lots de graines commerciaux issus de peuplements naturels, puis, à partir de 1966, de lots de graines récoltés par des scientifiques de l'INRA (Station d'amélioration des arbres forestiers) ou d'autres instituts de recherche. Ces dispositifs ont été utilisés pour sélectionner les provenances de Douglas les plus intéressantes, pour des critères d'adaptation aux conditions de milieu (taux de survie, tardiveté du débourrement, polycyclisme), de production (hauteur principalement, mais aussi circonférence et volume), de forme (branchaison, fourchaison, flexuosité) et de qualité du bois (densité moyenne, angle des fibres, homogénéité de la répartition de densité) (Jarret 1978, Rosette, 1986).

Certains dispositifs, parmi les premiers installés par l'INRA, sont maintenant suffisamment âgés pour permettre, à partir des hauteurs totales mesurées chaque année, les actions suivantes:

- construction de courbes de croissance hauteur-âge ;

- étude de l'évolution du classement sur la hauteur de quelques provenances ;

- mise en évidence de différences entre provenances au niveau stratégies de croissance en hauteur.

L'évolution du classement des provenances est particulièrement intéressante après 10 ans. On considère parfois cet âge comme raisonnable pour l'analyse des dispositifs de comparaison de provenances non mono-arbres : on estime souvent que le classement de provenances n'évolue plus notablement après 10 ans (Kleinschmit et al, 1987).
Ce type d'étude se multiplie aujourd'hui (voir par exemple Sprintz et al, 1989 sur Pinus taeda, ou Magnussen et Park, 1991. sur Larix kaempferi).

Deux grands types de méthodes d'analyse ont été utilisés dans cette étude : la modélisation des courbes de croissance et des analyses multivariables. On souhaite montrer comment des méthodes d'analyse assez faciles à mettre en œuvre permettent d'affiner le choix des provenances les mieux adaptées à des contraintes environnementales et sylvicoles définies.

On a choisi de ne pas traiter ici d'aspects purement génétiques, qui pourraient faire l'objet d'un autre article.

\section{MATÉRIEL ET MÉTHODES}

\section{Le matériel végétal}

Le matériel végétal est constitué de 25 lots de graines de Douglas récoltés par les marchands grainiers des États-Unis dans l'aire naturelle. La zone dont elles sont originaires va de l'île de Vancouver (Colombie Britannique, Canada) au nord, à l'Orégon (États-Unis) au sud, et de la côte Pacifique à l'est au versant est de la chaîne des Cascades à l'ouest (Jarret, 1978 ; Rosette, 1986).

\section{Le dispositif}

Le dispositif 1702 de comparaison de provenances commerciales de Douglas a été planté par I'INRA (département des recherches forestières, Station d'amélioration des arbres forestiers) à Saint-Julien-le-Petit, Haute-Vienne (ouest du Massif central) en avril 1965. Les plants avaient été élevés jusqu'à l'âge de 3 ans $(1+2)$ dans la pépinière d'Amance, près de Nancy (Meurthe-et-Moselle).

Les caractéristiques du lieu de plantation sont présentées dans le tableau I. Le dispositif expérimental est un lattice équilibré, constitué de 6 répétitions et de 30 blocs comprenant cha- 
Tableau I. Caractéristiques du lieu de plantation.

\begin{tabular}{lccccccc}
\hline $\begin{array}{l}\text { Altitude } \\
(m)\end{array}$ & Longitude & Latitude & $\begin{array}{c}\text { Température } \\
\text { annuelle }\left({ }^{\circ} \mathrm{C}\right)\end{array}$ & & $\begin{array}{c}\text { Pluviométrie } \\
\text { annuelle }(\mathrm{mm})\end{array}$ \\
\cline { 3 - 6 } \cline { 5 - 6 } & & & $1959-1983$ & $1977-1983$ & & $1959-1983$ & $1977-1983$ \\
\hline 450 & $1^{\circ} 44^{\prime} \mathrm{E}$ & $45^{\circ} 49^{\prime} \mathrm{N}$ & 9,3 & 9,4 & & 1307 & 1398 \\
\hline
\end{tabular}

cun 5 provenances. Chaque parcelle est constituée de 56 individus plantés à $2,5 \mathrm{~m}$ au carré (1600 plants/ha). Une première éclaircie systématique a eu lieu durant l'hiver 1979-1980 (18 ans depuis la graine). Elle a éliminé une ligne sur 7 et une diagonale sur 4, laissant environ 1050 arbres/ha sur pied. On a ainsi récolté environ $385 \mathrm{~m}^{3}$ (d'après Birot et Lanares, 1980).

Une seconde éclaircie systématique a eu lieu au printemps 1987 (25 ans depuis la graine), au cours de laquelle a été abattue une diagonale sur 3, laissant environ 700 arbres sur pied/ha.

\section{Échantillon et mesures}

Parmi les 25 provenances, et pour limiter le coût de l'opération, 12 ont été choisies pour représenter toute la gamme des comportements pour la croissance en hauteur.

Le tableau II présente la liste des provenances retenues, avec quelques-unes des caractéristiques de leur lieu d'origine. Pour chaque provenance, 2 arbres dominants de chacune des répétitions ont été choisis, abattus et mesurés ; soit 12 arbres par provenance et 144 arbres en tout. Les mesures ont eu lieu au printemps 1987, sur une partie des arbres abattus lors de cette éclaircie. II s'agit de la hauteur totale annuelle de 1 à 25 ans, mesurée rétrospectivement. À cause de la difficulté qu'il y avait à retrouver certaines limites entre pousses annuelles, il manque un certain nombre de données entre 1 et 5 ans. Des mesures ont été effectuées à 16 et à 25 ans sur un autre échantillon d'arbres du même dispositif, choisis non pas dominants, mais représentatifs de chaque provenance (Jarret, 1978 pour les mesures à 16 ans). Une trentaine d'arbres de chaque provenance, sans statut particulier dans le peuplement, ont été tirés au hasard et mesurés. Ces mesures ont été utilisées pour comparer les classements des 12 provenances obtenus à ces âges à l'aide de ces 2 types d'échantillons.

La hauteur d'un arbre en peuplement résulte de l'effet du génotype et de l'environnement. Parmi les composantes de l'environnement, la concurrence intervient davantage pour des arbres tirés au hasard que pour des arbres dominants : la croissance individuelle en hauteur est réduite quand la compétition est forte (Ottorini, 1991).

\section{Méthodes d'analyse}

\section{Modélisation de la croissance en hauteur}

L'autocorrélation provient de l'absence d'indépendance entre les mesures successives sur un même individu. L'hétéroscédascité est la nonhomogénéité de la variance dans les échantillons. Dans le cas de la plupart des observations, elle est liée à la taille, et augmente au cours du temps.

La non-prise en compte de l'autocorrélation et de l'hétéroscédascité lors de la comparaison, à l'aide de techniques statistiques paramétriques, des paramètres de courbes de croissance, peut invalider les résultats obtenus (Magnussen et Park, 1991).

Après examen visuel des graphiques hauteur-âge des arbres échantillons de chacune des 12 provenances, 2 modèles principaux ont été testés : un modèle linéaire et un modèle non linéaire. 
Tableau II. Localisation et quelques caractéristiques des peuplements où ont été récoltées les 12 provenances étudiées (d'après Rosette, 1986).

\begin{tabular}{rllrrrr}
\hline Code & Code INRA & Nom & $\begin{array}{c}\text { Altitude } \\
(\mathrm{m})\end{array}$ & $\begin{array}{c}\text { Latitude } \\
\text { Nord }\end{array}$ & $\begin{array}{c}\text { Longitude } \\
\text { Ouest }\end{array}$ & $\begin{array}{c}\text { Précipitations } \\
\text { annuelles }(\mathrm{mm})\end{array}$ \\
\hline & & & & & & \\
1 & ASHF1 & Ashford 1 & 458 & $46^{\circ} 48^{\prime}$ & $123^{\circ}$ & 1900 \\
2 & CA-LA & Cameron Lake & 200 & $49^{\circ} 15^{\prime}$ & $124^{\circ} 40^{\prime}$ & 1700 \\
3 & DARR2 & Darrington 2 & 520 & $48^{\circ} 10^{\prime}$ & $121^{\circ} 35^{\prime}$ & 1900 \\
4 & GLAC & Glacier & 670 & $48^{\circ} 50^{\prime}$ & $122^{\circ}$ & 1400 \\
5 & GR-FA & Granite Falls & 520 & $48^{\circ} 05^{\prime}$ & $122^{\circ}$ & 1500 \\
6 & HUMP & Humtulips & 50 & $47^{\circ} 12^{\prime}$ & $123^{\circ} 55^{\prime}$ & 1550 \\
7 & MA-CR & Marion Creek & 875 & $44^{\circ} 35^{\prime}$ & $121^{\circ} 55^{\prime}$ & 1750 \\
8 & MOLA & Molalla & 250 & $45^{\circ} 15^{\prime}$ & $122^{\circ} 25^{\prime}$ & 1025 \\
9 & NANA & Nanaimo & 40 & $49^{\circ} 10^{\prime}$ & $124^{\circ}$ & 1000 \\
10 & SANT & Santiam & 1100 & $44^{\circ} 27^{\prime}$ & $121^{\circ} 58^{\prime}$ & - \\
11 & SHEL & Shelton & 100 & $47^{\circ} 13^{\prime}$ & $123^{\circ} 10^{\prime}$ & - \\
12 & SKYK & Skykomish & 650 & $47^{\circ} 44^{\prime}$ & $121^{\circ} 13^{\prime}$ & 1900 \\
& & & & & & \\
\hline
\end{tabular}

\section{Le modèle linéaire}

Retrouver les limites entre les premiers accroissements annuels en hauteur sur des Douglas âgés de 25 ans présente des difficultés : des données manquent entre 1 et 5 ans. Cette période comprend l'élevage des plants en pépinière ( 1 an de semis et 2 ans de repiquage) et leur installation en forêt.

Si on tronque le fichier des données en lui retirant, pour chacune des provenances, les mesures des 5 premières années, on obtient des nuages de points hauteur-âge auxquels s'ajustent très bien des modèles linéaires de la forme $\mathrm{H}=\mathrm{a}+\mathrm{bt}$, où $\mathrm{H}=$ hauteur totale en $\mathrm{cm}, \mathrm{t}$ = âge en année depuis la graine, $\mathbf{a}=$ ordonnée à l'origine, $\mathrm{b}=$ pente de la droite.

On a estimé les paramètres du modèle linéaire pour chacun des 144 arbres des 12 provenances. II est ainsi possible de comparer les 12 provenances entre elles pour les paramètres suivants : pente de la droite et ordonnée de la droite à l'origine. Calculer des modèles individuels antre par arbre (plutót que des modèles globaux par provenance) évite d'avoir à tenir compte d'une éventuelle hétéroscédascité.

La validité des 144 modèles a été testée par |'examen des statistiques d'ajustement du modèle : coefficient de détermination $\left(R^{2}\right)$, probabi- lité pour que les paramètres des droites soient significativement différents de zéro, et résidus : les graphes des résidus en fonction du temps font apparaître, pour la plupart des modèles calculés, une autocorrélation entre résidus (Tornassone et al, 1983) ; d'après ces auteurs, on ne peut en rester là, et il est nécessaire d'améliorer les modèles :

La méthode employée est celle décrite par Tomassone (Tomassone et al, 1983), adaptée à l'étude d'une croissance annuelle :

soit le modèle :

$$
H_{i}=a+b t_{i}+u_{i}
$$

où les $u_{i}$ sont les résidus.

Si les résidus sont corrélés, une façon de l'écrire peut être $u_{i}=\alpha u_{i-1}+v_{i}$, où les $v_{i}$ satisfont aux conditions du modèle linéaire ; $\alpha$ n'est pas connu, mais peut être estimé. Une valeur élevée de l'estimation de $\alpha$ (proche de 1) traduit l'existence d'une autocorrélation entre les résidus.

Le modèle [1] peut alors s'écrire : $H_{i}-\alpha H_{i-1}=$ $a-\alpha a+b t_{i}-\alpha b t_{i-1}+u_{i}-\alpha u_{i-1}$, c'est-à-dire :

$$
H_{i}-\alpha H_{i-1}=a(1-\alpha)+b\left(t_{i}-\alpha t_{i-1}\right)+v_{i}
$$

où les $v_{i}$ satisfont aux conditions du modèle linéaire. 
Or ici $t_{i}=t_{i-1}+1$, puisque la variable explicative de nos modèles est le temps, exprimé en années, les dates étant régulièrement espacées d'un an. [2] s'écrit alors :

$$
H_{i}=a(1-\alpha)+b \alpha+\alpha H_{i-1}+b(1-\alpha) t_{i}+v_{i}
$$

qui est l'expression d'un modèle linéaire multiple $H_{i}=f\left(H_{i-1}, t_{i}\right)$ dont les coefficients sont :

- constante $=a(1-\alpha)+b \alpha$;

- coefficient de $\mathrm{H}_{\mathrm{i}-1}=\alpha$;

- coefficient de $t_{i}=b(1-\alpha)$.

L'estimation des coefficients du modèle (3) nous donne donc directement l'estimation de $\alpha$, assortie de la probabilité pour que cette estimation soit significativement différente de 0 , et permet de calculer, en cas d'autocorrélation significative, une estimation améliorée du coefficient $b$ du modèle [1] : $b=\left(\right.$ coefficient de $\left.t_{j}\right) /(1-\alpha)$

Pour estimer les coefficients de (3), on emploie le module de régression multiple de Statgraphics v.5, qui utilise la décomposition de Gram-Schmidt (Anonyme, 1991). Pour éviter d'avoir à formuler des hypothèses contestables ou difficilement vérifiables sur les fonctions de distribution des pentes des droites et des ordonnées a l'origine, on utilise pour comparer les provenances entre elles une technique non paramétrique, la méthode de Kruskall-Wallis, mise en œuvre à l'aide du logiciel Statgraphics. Ses hypotheses sont au nombre de 4 (Conover 1980 ; Sprent, 1989). Les 3 premières, à savoir : 1) il doit y avoir indépendance entre individus dans les échantillons, et entre échantillons ; 2) l'échelle de mesure est au moins ordinale ; 3) toutes les fonctions de distribution des populations sont identiques, ou bien certaines populations tendent à croître plus vite que d'autres, sont satisfaites sans probleme.

La $4^{e}$ hypothèse est la suivante : tous les échantillons sont des échantillons choisis au hasard dans leur populations respectives. Elle n'est satisfaite qu'à condition que l'on considère que les arbres-échantillon ont été tirés au sort dans des populations constituées des arbres dominants de chacune des provenances.

\section{Le modèle non linéaire}

Il est basé sur la fonction de Richards à 4 ou 3 paramètres.

Si on choisit de conserver les données des années 1 à 5 depuis la graine, la fonction de $\mathrm{Ri}$ - chards s'ajuste très bien aux nuages de points, et en particulier un petit peu mieux que le modèle linéaire.

La fonction de Richards est une expression de la forme $H=A\left(1-e^{(b-k t)}\right)^{n}$ où $H=$ hauteur totale $(e n c m$ ), $t$ = áge depuis le semis (en années), A est la valeur de l'asymptote de la courbe, $b, k$ et $n$ sont des paramètres caractérisant la forme de la courbe, mais sans signification biologique évidente (Causton et Venus, 1981).

\section{Évolution du classement sur la hauteur totale}

\section{Moyennes et médianes}

Chaque fois qu'on a voulu caractériser les provenances avec un paramètre unique, on a choisi de travailler à l'aide des hauteurs médianes plutôt que des hauteurs moyennes : on peut alors s'abstenir d'éliminer des échantillons des arbres à comportement anormal, qui auraient fortement affecté la moyenne, et auxquels la médiane est peu sensible. On évite ainsi de diminuer la taille d'échantillons déjà faibles (12 arbres par provenance).

Notons que la valeur du coefficient de corré lation linéaire entre moyennes et médianes des données, toutes provenances confondues, entre 6 et 25 ans, est de 0,93 .

\section{Evolution générale du classement sur la hauteur totale}

La technique de Classification Automatique proposée par le logiciel Unistat (V.3.0) a été utilisée pour définir des groupes de provenances durant les périodes 6-10 ans, 13-17 ans et 21-25 ans. Durant chacune des 3 périodes, le nombre de groupes a été fixé arbitrairement à 3 : bons, moyens et faibles pour la croissance en hauteur. Les limites entre ces groupes ont partout la même signification. L'algorithme utilisé pour calculer les distances entre individus et les regrouper est une version spéciale de l'algorithme de Ward nommée Reciprocal Neighbours Method (Anonyme, 1991). Les individus (lignes) des tableaux de données traités sont les 12 provenances, les variables (colonnes) sont les âges, et les données les médianes des hauteurs par provenance aux åges considérés. Les données ont été standardisées (Anonyme, 1991), de 
façon à ne pas accroître l'importance des variables quand l'âge augmente.

\section{Stratégies d'accroissement en hauteur}

Une année particulièrement sèche, 1976 (15 ans depuis la graine), a été retenue afin d'étudier si des stratégies particulières d'accroissement en hauteur existent entre provenances.

L'étude de son effet sur la croissance des 12 provenances s'est faite à l'aide de graphiques montrant l'évolution en fonction du temps des accroissements médians annuels en hauteur, toutes provenances confondues, et provenance par provenance. Ensuite, ces données ont été traitées à l'aide d'une classification automatique, puis d'une analyse en composantes principales (ACP).

La classification automatique a servi à classer les accroissements annuels en groupes homogènes. Le tableau traité comprend en lignes (individus) les 20 accroissements annuels, de 1968 à 1987, et en colonnes (variables) les 12 provenances. Les données sont les médianes des accroissements annuels. Les données n'ont pas été standardisées puisque toutes les variables sont de même nature et qu'on ne souhaite pas éliminer l'effet inter-années liè à la forme de la courbe de croissance.

L'ACP a servi à étudier le comportement des 12 provenances durant ces 20 années. Le tableau traité est le méme que le précédent, mais transposé (données non standardisées).

Puis une autre classification ascendante hiérarchique a été pratiquée sur ce dernier tableau de données. Logiciel Unistat, distance : Reciprocal Neighbours Method, données non standardisées. Elle propose des regroupements des provenances à profils ressemblants.

\section{RÉSULTATS}

\section{Arbres dominants et échantillon représentatif}

Les coefficients de corrélation linéaires entre hauteurs totales des arbres des échantillons dits dominants et représenta- tifs sont de 0,83 à 16 ans et 0,89 à 25 ans (et sont significativement différents de zéro à un pour mille). Ces coefficients de corrélation élevés montrent qu'il y a peu de différences de classement entre provenances, que l'échantillon soit dominant ou représentatif.

Les rares changements de classement sont tous de faible amplitude : les meilleures provenances restent les meilleures, les plus mauvaises restent les plus mauvaises quel que soit l'échantillon.

Donc le choix d'un échantillon d'arbres dominants ne modifie pas notablement le classement des provenances obtenu à l'aide d'un échantillon plus représentatif a priori.

Toutefois, à 16 ans comme à 25 ans, les seules modifications de classement interviennent à l'intérieur du groupe de tête, et concernent en particulier la provenance la mieux placée : à 16 ans, la provenance 5 (Granite Falls) passe de la $1^{\text {re }}$ position du classement dominants à la $5^{\mathrm{e}}$ position du classement représentatif. Elle échange sa position avec la provenance 8 (Molalla), qui passe de la $5^{e}$ place du classement dominants à la $1^{\text {re }}$ place du classement représentatif. À 25 ans, Granite Falls passe de la $2^{\mathrm{e}}$ place du classement dominants à la $6^{\mathrm{e}}$ place du classement représentatif.

\section{Performances générales}

Le tableau III présente les performances moyennes des 2 meilleures provenances à 25 ans, et toutes provenances confondues, comparées aux données des tables de production de Decourt (Douglas, ouest du Massif central, arbres dominants, Decourt et Vanniere, 1984) : le dispositif est donc installé dans une bonne station à Douglas (les classes 1 et 2 de Decourt correspondent respectivement à des productivités d'environ 17 et $\left.14 \mathrm{~m}^{3 /} / \mathrm{ha} / \mathrm{an}\right)$. À 
Tableau III. Comparaison des performances moyennes de 2 (la meilleure et la moins performante) des 12 provenances étudiées, avec celles d'un peuplement de Douglas installé dans des situations de classe 1 et 2 de l'ouest du Massif central (d'après Decourt et Vannière, 1984).

\begin{tabular}{|c|c|c|c|c|c|c|}
\hline & Toutes prov conf & $\begin{array}{l}\text { Marion Creek } \\
\text { (7) }\end{array}$ & $\begin{array}{l}\text { Shelton } \\
\text { (11) }\end{array}$ & $\begin{array}{l}\text { Table de } \\
\text { classe } 1\end{array}$ & $\begin{array}{l}\text { de Décourt } \\
\text { classe } 2\end{array}$ & $\begin{array}{l}\text { Production } \\
\text { classe } 3\end{array}$ \\
\hline $\begin{array}{l}\text { Hauteur totale } \\
\text { moyenne }(m)\end{array}$ & 18,9 & 17,0 & 20,1 & 20,6 & 18,2 & 15,6 \\
\hline
\end{tabular}

25 ans, les circonférences moyennes des provenances, calculées sur un échantillon représentatif d'une trentaine d'arbres par provenance, varient de 54 à $67 \mathrm{~cm}$ (pas de différence significative entre provenances).

\section{Modélisation de la croissance en hauteur}

\section{Ajustement linéaire}

Le tableau IV présente les résultats par provenance. II donne les médianes des coefficients des régressions linéaires, corrigées de l'autocorrélation entre les résidus, calculées sur chacun des 144 arbres.

Pour 28 arbres, la valeur estimée pour $\alpha$, coefficient d'autocorrélation entre résidus et résidus précédents, n'est pas significativement différente de 0 . L'autocorrélation significative des autres arbres est de 2 sortes : pour 12 arbres, la forme du nuage de points hauteur-âge est trop sigmoïdale pour être approximée à l'aide d'une droite. Ils ont été éliminés de l'échantillon. Pour les 104 autres arbres, l'examen des graphiques résidus/âge montre qu'il s'agit d'une autocorrélation imputable à des accidents de croissance du type cime cassée ou, surtout, ralentissement de la croissance dû à une diminution de la fertilité du milieu suite, par exemple, à une sécheresse exceptionnelle. Pour ces arbres, une autre façon d'éliminer l'autocorrélation est d'estimer un modèle pour chaque intervalle de temps précédent et suivant l'accident (Pichot, communication personnelle). Toutefois, cette technique présente l'inconvénient de rendre la comparaison des modèles moins facile.

Les valeurs des $R^{2}$ (pourcentage de la variance expliquée par les modèles) sont

Tableau IV. Médianes par provenance des pentes corrigées et ordonnées à l'origine des droites de régression des 144 arbres de l'échantillon.

\begin{tabular}{cc} 
Provenances & $\begin{array}{c}\text { Ordonnée Pente Rang } \\
\text { à l'origine (médiane) } \\
\text { (médiane) }\end{array}$ \\
\hline
\end{tabular}

$\begin{array}{lrrr}\text { 1 Ashford 1 } & -343 & 94,99 & 4 \\ \text { 2 Cameron lake } & -416 & 93,06 & 5 \\ \text { 3 Darrington 2 } & -401 & 96,00 & 2 \\ \text { 4 Glacier } & -360 & 91,93 & 6 \\ \text { 5 Granite falls } & -341 & 89,85 & 9 \\ \text { 6 Humptulips } & -459 & 99,25 & 1 \\ \text { 7 Marion creek } & -328 & 76,44 & 12 \\ \text { 8 Molalla } & -362 & 88,25 & 10 \\ \text { 9 Nanaimo } & -386 & 91,20 & 7 \\ \text { 10 Santiam } & -377 & 85,29 & 11 \\ \text { 11 Shelton } & -416 & 95,52 & 3 \\ \text { 12 Skykomish } & -362 & 90,94 & 8\end{array}$


toujours supérieures à 0,99 , et tendent à montrer que les droites calculées sont, pendant la période considérée (c'est-à-dire entre 6 et 25 ans), de bons modèles de la croissance des arbres.

La pente des droites résume la vitesse de croissance en hauteur des arbres entre 6 et 25 ans : un classement des provenances selon la médiane des pentes met en évidence la vigueur des provenances 11 (Shelton), 6 (Humptulips) et 3 (Darrington 2) pendant la période considérée.

L'analyse de Kruskall-Wallis met en évidence l'existence de différences significatives entre provenances sur le paramètre estimé pente de la droite de régression. Les résultats sont présentés dans le tableau $V$. Notons que les classements des provenances selon la médiane des pentes des droites de régression (tableau IV), d'une part, et selon le rang moyen des

Tableau V. Résultat du test de comparaison des 12 provenances pour le facteur pente des droites de régression, à l'aide de la méthode de Kruskall-Wallis. Les crochets regroupent les provenances non significativement différentes. Les rangs sont les rangs moyens des arbres de la provenance.

\begin{tabular}{|c|c|c|}
\hline Provenances & $\begin{array}{l}\text { Rang } \\
\text { moyen }\end{array}$ & $\begin{array}{c}\text { Groupes } \\
\text { homogenes }\end{array}$ \\
\hline $\begin{array}{l}10 \text { Santiam } \\
7 \text { Marion creek } \\
9 \text { Nanaimo } \\
8 \text { Molalla } \\
12 \text { Skykomish } \\
5 \text { Granite falls } \\
2 \text { Cameron lake } \\
4 \text { Glacier } \\
11 \text { Shelton } \\
3 \text { Darrington } 2 \\
1 \text { Ashford } 1 \\
6 \text { Humptulips }\end{array}$ & $\begin{array}{l}34,92 \\
39,63 \\
56,83 \\
56,90 \\
58,00 \\
58,00 \\
69,55 \\
70,00 \\
81,67 \\
85,91 \\
83,92 \\
97,80\end{array}$ & $\perp$ \\
\hline
\end{tabular}

arbres de chaque provenance classés sur cette même pente (tableau V), d'autre part, sont légèrement différents (coefficient de corrélation linéaire : 0,90 ). Le groupe des provenances les plus vigoureuses est composé des provenances 6 (Humptulips), 1 (Ashford 1), 11 (Shelton), 3 (Darrington 2) et 4 (Glacier). Il est complètement disjoint du groupe des provenances les moins vigoureuses : 5 (Granite falls), 12 (Skykomish), 8 (Molalla), 9 (Nanaimo), 7 (Marion creek) et 10 (Santiam).

Par contre, il n'existe pas de différence significative entre provenances pour le facteur ordonnée de la droite à l'origine.

\section{Ajustement non linéaire : la fonction de Richards}

L'estimation de modèles individuels acceptables (arbre par arbre) à l'aide de la fonction de Richards n'a pas été possible : les estimations proposées sont souvent de très mauvaise qualité (avec des écarts types sur l'estimation de la valeur des paramètres du même ordre de grandeur que la valeur du paramètre). Pour chaque provenance, trop d'arbres sont encore trop loin de la phase de ralentissement de croissance pour que la forme de la courbe puisse être correctement estimée.

Seuls des modèles construits à partir du nuage de points regroupant les hauteurs successives des 12 arbres de chaque provenance ont pu être efficacement calculés. Malheureusement, ils ne prennent en compte ni l'autocorrélation, ni l'hétéroscédascité des données : ils sont donc très probablement entachés de biais (Magnussen et Park, 1991). De plus, la précision de l'estimation de certains paramètres, comme l'asymptote des courbes de croissance, est faible. Aucune technique de comparaison des paramètres des courbes de croissance n'a été mise en œuvre. 


\section{Evolution du classement sur la hauteur totale}

\section{Évolution générale}

Le tableau VI présente l'évolution de la composition des 3 groupes de provenances (bon, moyen, faible) définis durant les périodes 6-10 ans, 13-17 ans, 21-25 ans à l'aide d'une classification automatique.

Les provenances 4 (Glacier), 5 (Granite Falls), 7 (Marion Creek), 8 (Molalla), 9 (Nanaïmo), 10 (Santiam), 12 (Skykomish) ne changent jamais de groupe : leur position reste stable.

Quatre provenances voient leur position s'améliorer : la provenance 2 (Cameron Lake) passe, entre la période 13-17 ans et la période 21-25 ans, du groupe faible au groupe moyen, les provenances 3 (Darrington 2) et 11 (Shelton) passent, entre les mêmes périodes, du groupe moyen au groupe bon, et surtout la provenance 6 (Humptulips) passe, en 2 fois, du groupe faible au groupe bon. Sa vigueur, mise en évidence par sa première position dans les classements sur la vitesse moyenne de croissance, se traduit ici par la meilleure progression dans le classement sur la hauteur totale.
Seule la provenance 1 (Ashford) perd du terrain, passant du groupe bon de la période 6-10 ans au groupe moyen de la période 13-17 ans. Sept provenances restent stables, 4 voient leur position s'améliorer et une seule recule.

\section{Évolution du classement relatif des provenances}

La figure 1 présente l'évolution du classement relatif sur la hauteur des 12 provenances.

L'impression générale donnée par la classification en groupes est confirmée et précisée : la position des provenances 4 (Glacier), 5 (Granite Falls), 7 (Marion Creek), 8 (Molalla), 9 (Nanaïmo), 10 (Santiam), 12 (Skykomish) n'évolue guère dans le classement général. Toutefois, la provenance 5 (Granite Falls), après avoir largement et longuement occupé la tête du classement, est depuis l'âge de 23 ans talonnée par les provenances 3 (Darrington 2), 11 (Shelton) et surtout 6 (Humptulips), qui est remarquablement passée de la $9^{e}$ position à l'âge de 7 ans à la $4^{e}$ place depuis l'âge de 22 ans.

On distingue très bien la séparation, vers l'âge de 12 ans, de la provenance 2

Tableau VI. Évolution de la composition de 3 groupes de provenances (faibles, moyennes, bonnes pour la croissance en hauteur) pendant 3 périodes choisies (6-10 ans : début de croissance, 13-17 ans : pleine croissance, 21-25 ans : léger ralentissement de croissance).

Groupes

Composition des groupes

pendant la période considérée code provenance)
6 a 10 ans
13 à 17 ans
21 à 25 ans

$\begin{array}{llll}\text { Bon } & 1,5 & 5 & 3,11,6,5 \\ \text { Moyen } & 3,8,11,4,12,9 & 1,8,3,11,4,12,6,9 & 1,8,4,12,2,9 \\ \text { Mauvais } & 2,10,6,7 & 2,7,10 & 7,10\end{array}$




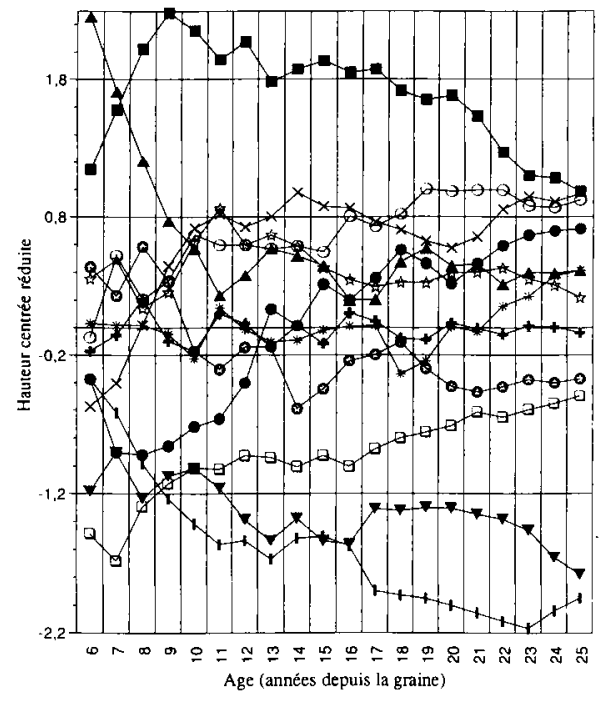

Fig 1. Evolution du classement relatif sur la hauteur des 12 provenances entre 6 et 25 ans. $-\mathbf{A}-1$ Ashford 1 ; $-\square-2$ Cameron lake; O-3 Darrington 2; - -4 Glacier; -5 Granite falls; $\longrightarrow-6$ Humptulips; $-1-7$ Marion creek; - 1 -8 Molalla; - -9 Nanaimo; -10 Santiam; -X-11 Shelton; - -12 Skykomish.

(Cameron Lake) du groupe de queue constitué par les provenances 7 et 10 .

Entre 6 et 11 ans la provenance 1 (Ashford) s'effondre et passe de la $1^{\text {re }}$ à la $5^{\mathrm{e}}$ position. Elle conserve ensuite pratiquement toujours cette $5^{\mathrm{e}}$ place.

Une étude des classements relatifs des provenances basée uniquement sur l'évolution de leurs rangs ne permettrait pas, comme ici, de mettre en évidence des évolutions comme celles concernant les provenances 2 et 5, qui s'écartent l'une de l'autre en gardant le même rang.

\section{Prédiction des performances à 25 ans}

La figure 2 présente l'évolution de la valeur du coefficient de corrélation linéaire entre performances à 25 ans, et performances aux âges 6 à 24 ans depuis la graine, des provenances. Elle permet de repérer l'âge à partir duquel on aurait pu prédire correctement les performances à 25 ans des provenances.

Si on considère ensemble les 12 provenances, c'est entre 14 et 15 ans que le coefficient de corrélation dépasse définitivement la valeur 0,90 , limite arbitraire choisie ici pour une bonne prédiction du classement à 25 ans.

Si on retire du groupe des provenances les provenances 5 et 6 (Granite Falls et Humptulips), la valeur 0,90 du coefficient de corrélation est dépassée dès 12 ans : la présence des provenances 5 et 6 dans le groupe étudié recule de 3 ans la date à partir de laquelle le classement global peut être considéré comme un bon prédicteur du classement à 25 ans.

Si on retire du groupe des provenances les provenances 7 et 10 (Marion Creek et Santiam), la valeur 0,90 du coefficient de corrélation n'est dépassée qu'à partir de 21 ans : ces 2 provenances stabilisent fortement le classement général.

Kleinschmit (Kleinschmit et al, 1987) a étudié l'évolution des performances de 29 provenances de Douglas extraites de la collection IUFRO, installées sur 6 sites en Allemagne, de 3 à 14 ans, puis de 10 à 14 ans depuis la graine (soit entre 1972 et 1983, puis entre 1979 et 1983). II ne trouve que très peu de relation entre les performances à 3 ans (en pépinière) et les performances à 14 ans (coefficient de corrélation de 0,37 ). Par contre, très peu de modifications de rangs intervenaient entre 10 et 14 ans (coefficient de corrélation : 0,97 ). Il en conclut que "la hauteur de provenances peut être assez bien estimée à 10 ans". Ce jugement doit être nuancé, si I'on observe l'évolution du coefficient de corrélation entre les performances à 10 ans et les performances de 11 à 25 ans de 


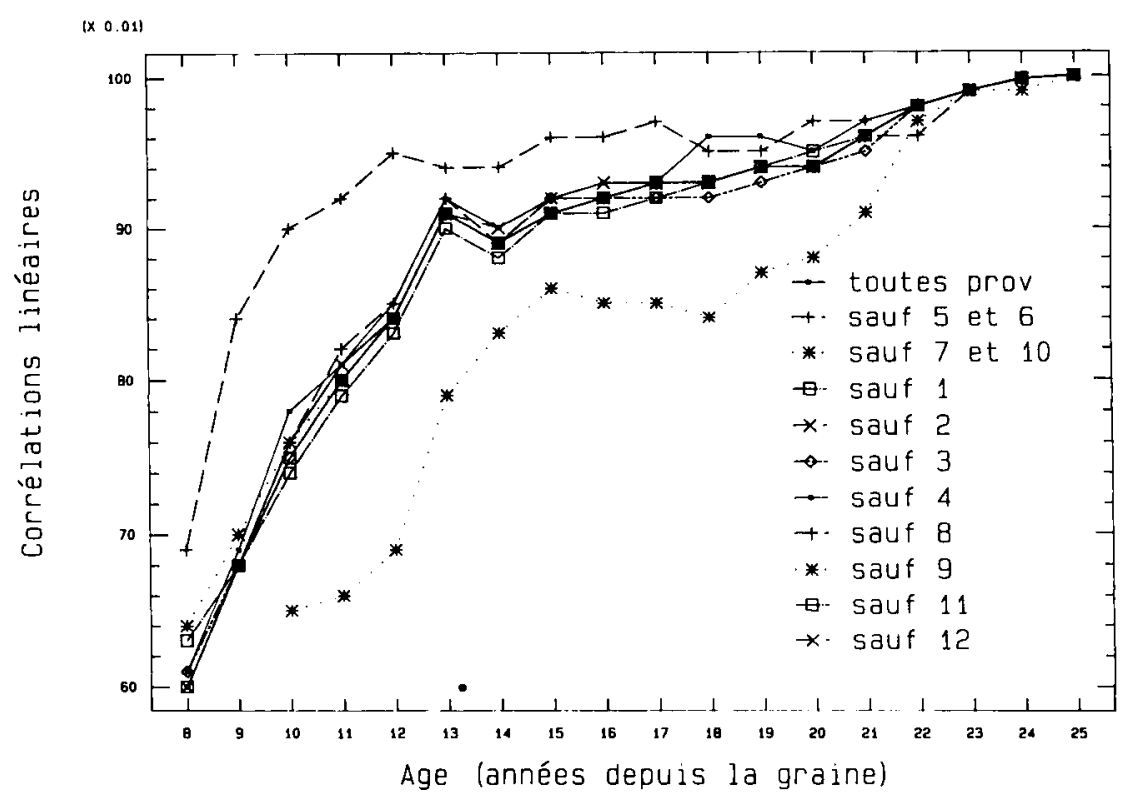

Fig 2. Evolution de la corrélation linéaire entre hauteurs médianes par provenance à 25 ans et hauteurs médianes par provenance entre 8 et 25 ans. Les valeurs des corrélations sont significativement différentes de $0 \%$.

notre lot de provenances (fig 3). Toutes provenances confondues, le coefficient de corrélation linéaire diminue régulièrement, atteignant la valeur 0,76 à 25 ans. Quelle sera sa valeur aux alentours de 50 ans? L'évolution actuelle semble indiquer qu'elle pourrait très bien être inférieure à 0,50.

Si on retire du lot de provenances étudiées Granite Falls et surtout Humptulips (provenances 5 et 6 ), le coefficient de corrélation diminue beaucoup plus lentement, puisqu'il est à peine inférieur à 0,90 à 25 ans. Par contre, si on retire les provenances Marion Creek et Santiam (7 et 10), le coefficient de corrélation diminue plus rapidement, jusqu'à 0,65 à 25 ans, confirmant le rôle de stabilisateurs des provenances Marion Creek et Santiam.

\section{Stratégies d'accroissement annuel en hauteur des provenances}

Des différences de comportement entre provenances, au niveau accroissements en hauteur, ont donc été mises en évidence. L'étude des profils des médianes des accroissements annuels en fonction du temps permet de bien mettre en évidence ces différences de comportement, et de proposer une typologie sommaire des stratégies d'accroissement des provenances sur le site de Saint-Julien-le-Petit.

\section{L'année 1976}

Le graphique des médianes des accroissements annuels en fonction du temps, 


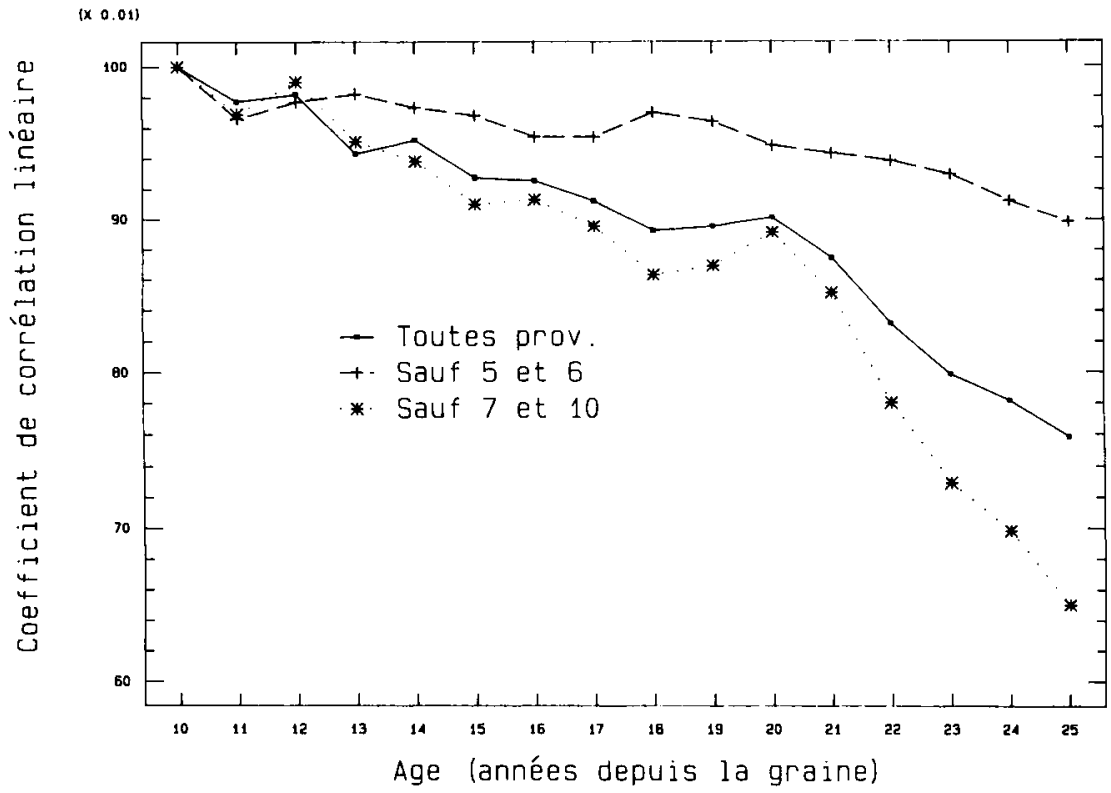

Fig 3. Évolution des corrélations linéaires entre hauteurs médianes par provenance à 10 ans et hauteurs médianes par provenance entre 10 et 25 ans. Les valeurs des corrélations sont significativement differentes de $0 \%$.

toutes provenances confondues (fig 4), a une forme rappelant celle d'une dérivée de courbe de croissance (il comprend les années 1 à 6 , exclues de la modélisation; ce sont ces années qui contribuent le plus à donner cette forme à cette courbe) ; on peut y définir 3 phases de croissance : augmentation rapide (années 1 à 7 ) ; stabilisation à un niveau élevé (années 8 à 18 ou 19) ; diminution peu importante (années 19 ou 20 à 25).

II possède un creux particulièrement marqué au niveau de l'année 15 (1976), connue jusqu'à aujourd'hui, dans la mémoire collective française, comme "'année de la sécheresse». Les relevés de la station météorologique de Peyrat-leChâteau montrent un important déficit pluviométrique pendant la saison de végétation 1976, en particulier pendant les mois d'avril à juillet. Les précipitations des quelques années précédentes (en particulier de la précédente) et suivantes sont normales.

Sur les 5 années comprises entre 1974 et 1978, seule l'année 1976 (15) a donné lieu à une croissance anormalement faible. La figure 5 présente les 12 graphiques des accroissements médians annuels entre 6 et 25 ans, provenance par provenance. II n'y a pas plus de changement global de classement entre 1975 et 1977 qu'il n'y en a entre n'importe quel autre couple d'années séparées de 2 ans. Par contre, il est probable que plusieurs sécheresses successives ou simplement rapprochées, d'ampleur comparable à celle de 1976, aient une influence sur l'évolution du classement des provenances. L'étude des provenances à partir des modèles de crois- 


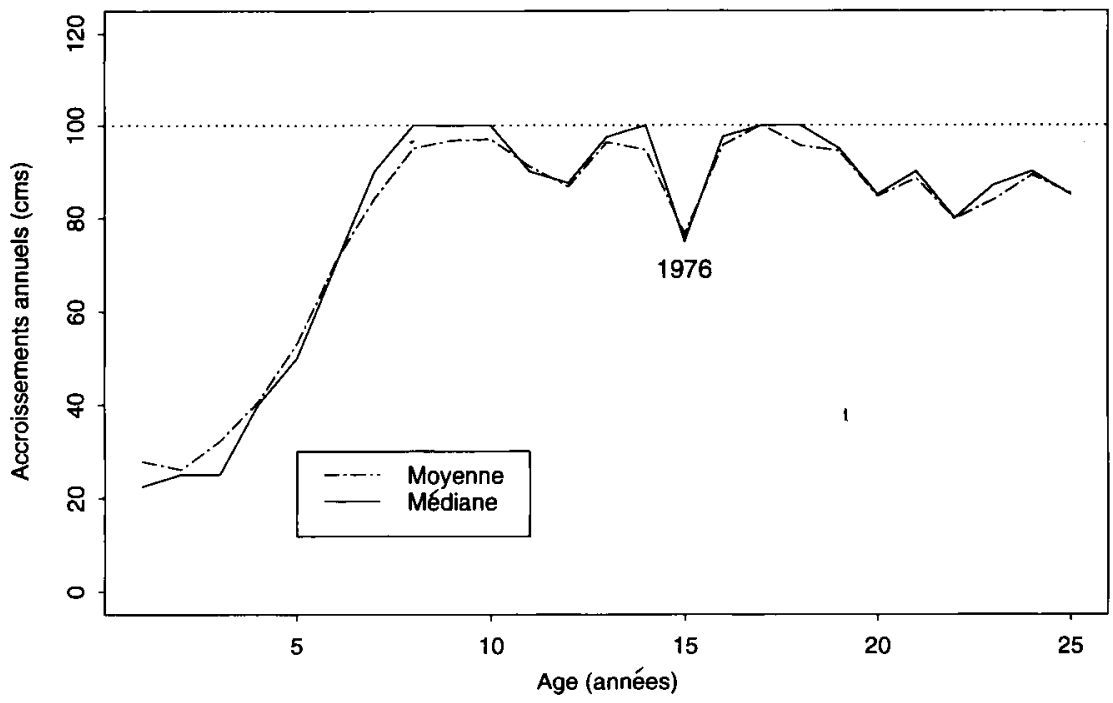

Fig 4. Médianes des accroissements annuels en hauteur, toutes provenances confondues.

sance calculées au paragraphe «Modélisation de la croissance en hauteur" ne permet pas de mettre en évidence l'effet de la sécheresse de 1976 sur la croissance des provenances.

Le fort effet 1976 visible sur les accroissement annuels toutes provenances confondues n'existe pas de façon évidente pour certaines provenances (fig 5). La typologie des provenances du paragraphe suivant met en évidence ces différences de comportement.

\section{Stratégies d'accroissements annuels : typologie des provenances}

Classification automatique des accroissements annuels

La troncature de l'arbre de la classification à un niveau proposant 4 groupes permet d'interpréter ces groupes assez facilement.
Un groupe contient les années à forts accroissements : 8 (1969), 9 (1970), 10 (1971), 13 (1974), 14 (1975), 16 (1977), 17 (1978), 18 (1979), 19 (1980).

Un deuxième groupe contient les années à faibles accroissements : 11 (1972), 12 (1973), 20 (1981), 21 (1982), 22 (1983), 23 (1984), 24 (1985), 25 (1986). Ce sont surtout les années 20 à 25 , où la croissance commence à ralentir, plus 2 années qui devraient normalement montrer une bonne croissance, mais qui possèdent en fait un déficit de croissance, toutefois plus faible que celui de 1976.

Un troisième contient des années à très faible accroissement : 6 (1967) et 15 (1976). L'accroissement de l'année 6 est encore un accroissement de début de croissance. Celui de l'année 15 , en pleine période de forte croissance, correspond à l'année sèche 1976.

Enfin, un dernier groupe contient une année originale : la 7 (1968). Selon les 

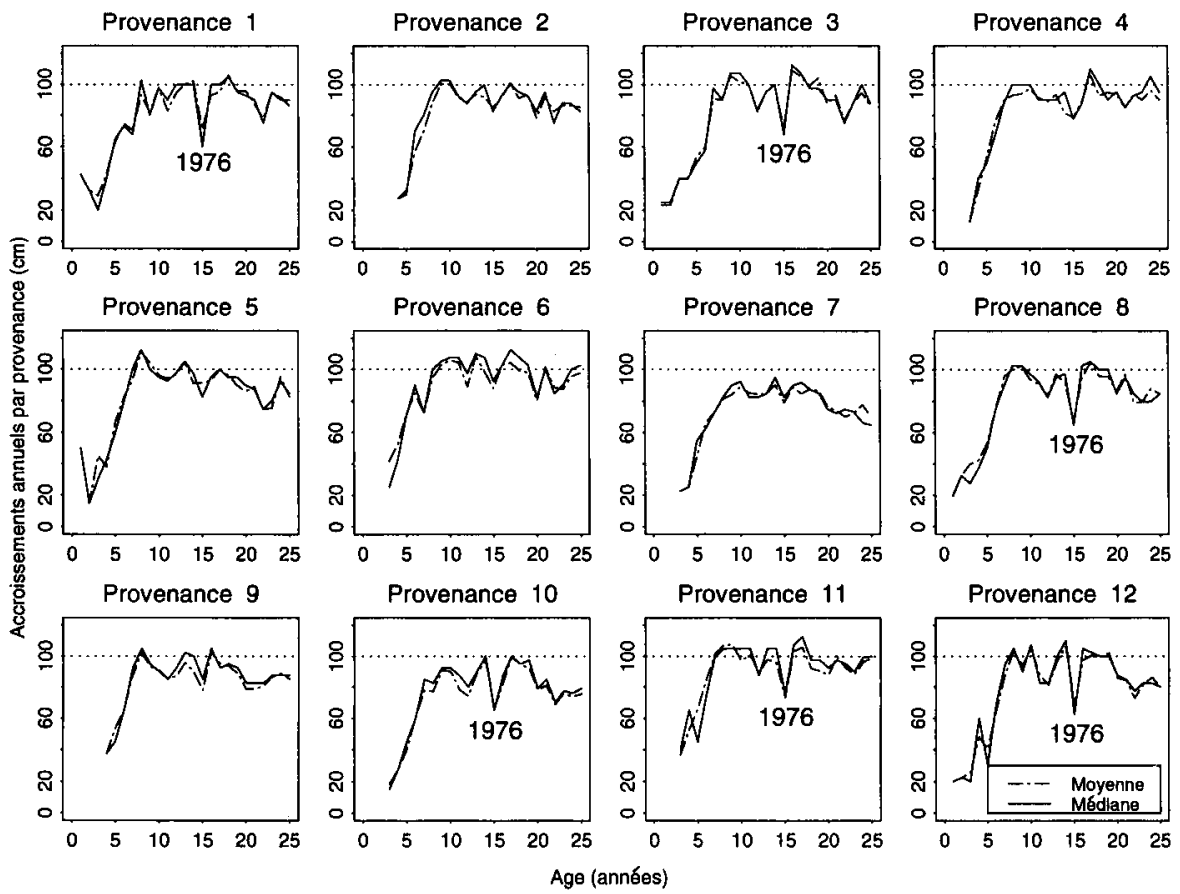

Fig 5. Médianes des accroissements annuels en hauteur, provenance par provenance.

provenances, c'est soit encore une année de début de croissance, à faible accroissement, soit déjà une année de pleine croissance, à fort accroissement.

\section{Analyse en composante principale des profils d'accroissement annuels médians des 12 provenances}

Le tableau d'ACP (tableau VII) montre que les 3 premières composantes principales expliquent $70 \%$ de la variabilité contenue dans les 20 variables initiales. En réduisant le nombre de variables initiales à 5 composantes principales, on arrive à expliquer $88 \%$ de la variabilité. II est inutile de considérer les composantes principales suivantes, qui expliquent moins de variabilité que n'en explique en moyenne cha-
Tableau VII. Tableau de l'analyse en composantes principales typologie des provenances. Cinq composantes principales résument bien la variabilité des 20 variables initiales.

\begin{tabular}{ccr}
\hline $\begin{array}{c}\text { No } \\
\text { composante }\end{array}$ & $\begin{array}{c}\text { Pourcentage } \\
\text { variance }\end{array}$ & $\begin{array}{c}\text { Pourcentage } \\
\text { cumule }\end{array}$ \\
\hline 1 & 44,19271 & 44,19271 \\
2 & 15,05914 & 59,25189 \\
3 & 11,10558 & 70,35443 \\
4 & 9,80616 & 80,16060 \\
5 & 8,17676 & 88,33736 \\
6 & 3,85474 & 92,19210 \\
7 & 2,86394 & 95,05603 \\
8 & 1,97116 & 97,02720 \\
9 & 1,39068 & 98,41788 \\
10 & 0,98080 & 99,39668 \\
11 & 0,60131 & 99,99998 \\
12 & 0,00001 & 100,00000 \\
\hline
\end{tabular}


cune des variables initiales (c'est-à-dire $5 \%)$.

Le classement des 12 provenances selon la composante 1 (fig 6) caractérise leur vigueur, en insistant particulièrement sur la vigueur à 24 et 25 ans. Remarquons que le coefficient de corrélation linéaire entre la position des 12 provenances sur la composante 1 et la médiane des pentes des droites de régression vaut 0,90 . Avec le rang moyen des provenances classées sur la pente des droites de régression, la valeur de ce coefficient de corrélation est de 0,85 .

Le classement des provenances selon la composante 2 (fig 6), à une exception près, s'effectue selon leur sensibilité à la sécheresse de 1976 (année 15) : seule Ashford 1 située, dans le plan des 2 premières composantes, près de provenances peu sensibles à 1976, est en fait très sensible à 1976. Mais l'examen de la figure 7 montre qu'Ashford 1 est en fait, dans le volume des 3 premières composantes, éloignée de Cameron Lake, Granite Falls, Glacier ou Nanaimo (peu sensibles à 1976), et relativement proche de Skykomish, Molalla et Darrington 2 (sensibles à 1976). Ashford 1 possède une particularité commune avec Humptulips, qui explique peut être leur position relativement proche dans le plan des 2 premières composantes : ces 2 provenances sont les seules à avoir un profil descendant entre les années 6 et 7 . Notons la position isolée des provenances Humptulips (6) et Marion Creek (7).

\section{Regroupements par types de profil}

La technique précédente met en évidence des ressemblances entre profils d'accroissements annuels médians, mais ne permet pas de définir aisément des groupes de provenances à profils comparables.

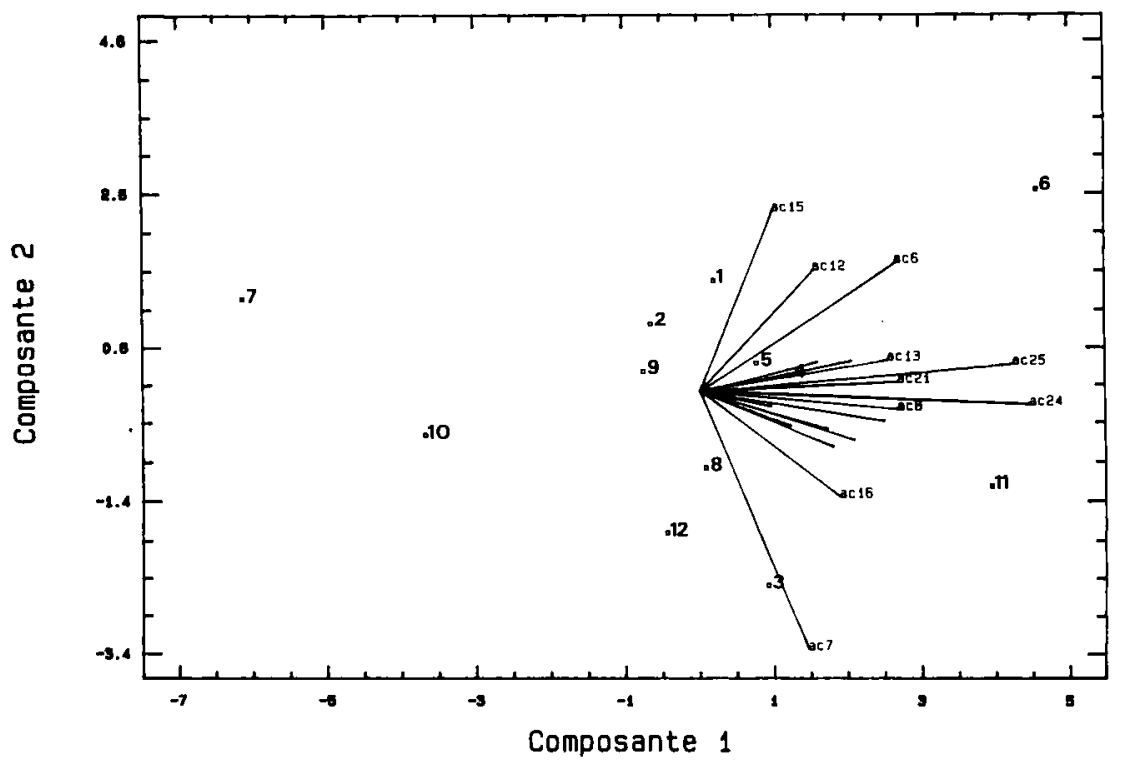

Fig 6. Les 20 variables (accroissements médians annuels) dans le plan des 2 premières composantes. Les points indiquent l'emplacement des provenances, repérées par leur numéro. AC15, etc, signifie accroissement de l'année 15. 


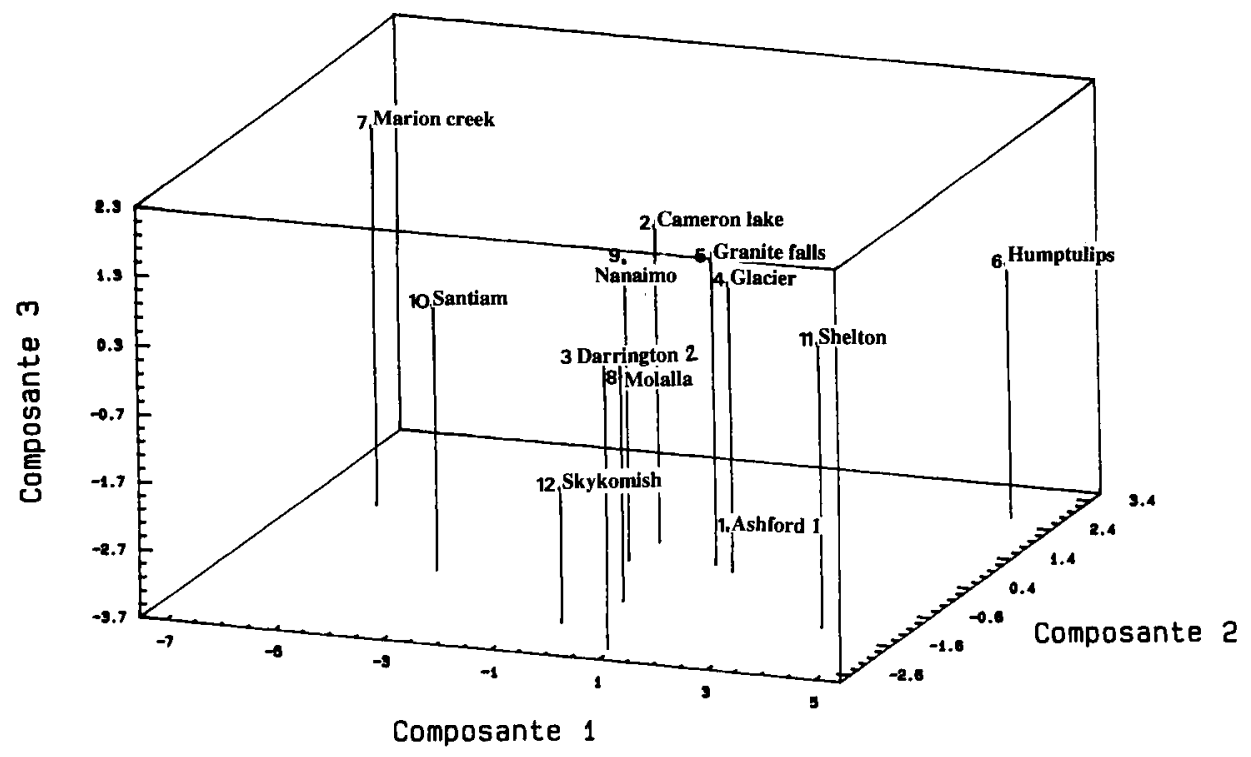

Fig 7. Emplacement des provenances dans le volume des 3 premières composantes.

La classification ascendante hiérarchique des 12 provenances, réalisée sur la base des mêmes données, propose des regroupements plutôt en fonction de la forme des profils, sans tenir compte d'une éventuelle superposition.

Selon le niveau où on coupe l'arbre de la classification, on fait varier le nombre de groupes, et donc leur composition. La troncature la plus intéressante propose 4 groupes, et isole 2 provenances :

- groupe $1: 4$ provenances (2, Cameron Lake, 8 , Molalla, 10, Santiam et 12, Skykomish) ;

- groupe 2 : provenances 9 (Nanaimo) et 5 (Granite Falls) ;

- groupe $3:$ provenances 1 (Ashford 1) et 4 (Glacier) ;

- groupe 4 : provenances 3 (Darrington 2) et 11 (Shelton).
Les 2 provenances isolées sont la 6 (Humptulips) et la 7 (Marion Creek).

Les ressemblances entre profils de provenances d'un même groupe peuvent s'apprécier sur la figure 5. La classification propose des groupes de profils de forme similaire, sans, ou presque, tenir compte des différences de vigueur, alors que I'ACP classe plutôt les provenances sur leur vigueur.

\section{DISCUSSION}

À quel âge peut-on considérer que le classement d'un lot de provenances est devenu stable ? On a vu (paragraphe "Prédiction des performances à 25 ans") que la réponse varie en fonction de la composition du lot de provenances étudié. Elle est également certainement fonction 
de l'intensité de sélection que l'on souhaite appliquer à la population de base. Plus la date de sélection est précoce, et plus l'intensité est forte, et plus on risque de laisser de côté des provenances se révélant tardivement, tout comme on risque de sélectionner des provenances s'essouflant vite. À 10 ans, une sélection sévère ne garderait que la provenance 5 (Granite Falls). Or, ce n'est pas forcément, sans même tenir compte du problème d'échantillonnage (voir paragraphe "Arbres dominants et échantillon représentatifm), le meilleur choix. Une sélection plus large laisserait certainement de côté la provenance 6 (Humptulips) au comportement original, du style coureur de fond, qui pourrait laisser penser qu'elle n'a pas fini de remonter des places au classement général.

Les résultats de cette étude peuvent aider à choisir, parmi les provenances étudiées, celle(s) qui conviendrai(en)t le mieux à un projet sylvicole donné.

Un type de sylviculture du Douglas couramment promu en France aujourd'hui consiste à planter à densité faible $(600$ à 1100 plants/ha), et à intensifier soins (dégagements) et élagages. Un tel peuplement devra être régulièrement et assez fortement dépressé et/ou éclairci, de façon à produire rapidement (45-50 ans dans les meilleures stations) des bois de qualité correcte pour usages nobles (voir par exemple Riou-Nivert, 1989 ; Armand et al, 1988).

Des qualités particulières demandées à une provenance plantée dans le cadre de cette sylviculture pourraient être, en plus de l'indispensable adaptation à la station : 1) une bonne croissance (de bonnes performances à 45-50 ans) ; 2) un démarrage rapide (pour économiser en dégagements) ; 3) une faible sensibilité aux accidents climatiques du type sécheresse.

La condition 1) entraîne, à défaut du classement à 45 ans, la sélection des pro- venances les plus performantes à 25 ans (fig 1) : $11,5,3,6,1$, si on se limite aux 5 premières.

Quatre de ces 5 provenances sont également parmi les 5 meilleures en ce qui concerne le classement sur la médiane des pentes de régression par provenance (tableau IV). Granite Falls est mal classée dans le tableau IV, car ses accroíssements d'entre 9 et 25 ans sont systématiquement inférieurs à ceux des autres provenances évoquées ci-dessus. Mais elle bénéficie jusqu'à 25 ans de la grande avance que lui a donné sa remarquable croissance entre 1 et 9 ans (fig 1).

La consultation du classement entre 6 et 10 ans permet d'éliminer les provenances 6 (Humptulips) et 11 (Shelton), à démarrage lent (condition 2) (fig 1).

Le classement des provenances en groupes plus ou moins sensibles à la sécheresse de 1976 permet d'éliminer encore les provenances 1 (Ashford 1) et 3 (Darrington 2) (paragraphe "Analyse en composante principale...", fig 5,7 ). La sélection ne comporte donc plus que Granite Falls (5).

Ce choix peut être modifié si on considère que la condition 3) ne se justifie pas, dans la mesure où la sécheresse de 1976 n'a pas particulièrement affecté le classement des provenances. II n'y a alors plus de raison d'éliminer de notre liste de présélectionnées les provenances 3 (Darrington 2) et 1 (Ashford 1). Parmi ces provenances, 2 se détachent nettement en ce qui concerne la condition 2) : Ashford 1 et Granite Falls. Un classement des provenances selon les seules pentes des droites (tableau IV) aurait donc été dangereux, puisqu'il aurait conduit à éliminer Granite Falls.

En revanche, pour une sylviculture ayant plutôt pour objectif de produire à longue révolution des bois de qualité, les critères de choix changeront. Des prove- 
nances comme Humptulips ou Shelton, croissant encore rapidement à 25 ans, semblent intéressantes. Une modélisation des courbes de croissance des arbres de ces provenances à l'aide par exemple de la fonction de Richards aurait fourni et permis de calculer des paramètres utiles, comme l'asymptote de la courbe de croissance, ou la durée de la période durant laquelle se fait la majorité de la croissance. Toutefois, 5 à 10 années de mesures supplémentaires auraient été nécessaires pour estimer ces paramètres avec une précision permettant de les comparer efficacement.

Sprinz et al (1989) disposaient de ces années supplémentaires et ont pu construire et comparer des modèles basés sur des fonctions du même type que celle de Richards. Ils ont mis en évidence des différences significatives entre provenances, et ont pu les classer sur leur vigueur. Ils n'ont pas pris en compte autocorrélation et hétéroscédacité. Magnussen et Park (1991) ont construit des modèles de croissance tenant compte de l'autocorrélation et de l'hétéroscédascité, et ont mis en évidence des groupes de provenances ayant même vitesse de croissance à l'aide d'une classification automatique sur les paramètres des modèles. Ils ont pris énormément de précautions pour que leurs modèles soient les meilleurs possibles. Pourtant, pour ces auteurs, «les modèles de croissance sont au mieux de grossières approximations d'un processus complexe». La modélisation oblige en effet à faire des choix a priori et masque des phénomènes intéressants, comme dans notre étude l'effet de la sécheresse de 1976. Pour comparer des stratégies de croissance, des traitements graphiques simples comme celui présenté sur la figure 1 , et des analyses multivariables comme I'ACP informent plus que la comparaison de modèles de croissance, tout en étant plus faciles à mettre en œuvre. ACP et classifica- tion automatique semblent complémentaires : ici, l'ACP a surtout permis de classer les provenances sur leur vigueur, et sur leur comportement lors d'années particulières, comme 1976. La classification automatique propose plutôt une synthèse du comportement relatif des provenances. Notons que les classements sur la vigueur obtenus grâce à la modélisation d'une part et à I'ACP d'autre part sont tout à fait comparables.

\section{CONCLUSION}

Cette étude a permis de mettre en évidence une partie de la richesse des informations que l'on peut déduire de séries de mesure aussi simples que la succession des hauteurs totales d'arbres de génotypes connus. Elle peut également aider à choisir une technique d'analyse de données de croissance adaptée à ses objectifs :

- la modélisation est plutôt destinée à "simplifier les multiples aspects de la croissance de provenances jusqu'à un degré bien adapté à la prise de décision" (Magnussen et Park, 1991) ;

- les analyses multivariables permettent de comparer les provenances non seulement pour leur vigueur globale, mais également pour d'autres aspects importants de leur comportement.

\section{REMERCIEMENTS}

Je souhaite remercier B Roman Amat et JC Bastien, qui m'ont confié cette étude, et m'ont guidé lors des premières étapes de ce travail ; $C$ Bastien, F Lefèvre et $C$ Pichot qui ont toujours été disponibles pour répondre à mes questions d'ordre statistique ; JM Ottorini, F Lefèvre et L Pâques, qui ont lu et commenté le manuscrit de cet article, avec une mention particulière pour $L$ Pâques qui a fait cet effort à plusieurs reprises, ainsi que les 2 lecteurs anonymes qui m'ont fait de nombreuses et importantes suggestions. 


\section{RÉFÉRENCES}

Anonyme (1991) Statgraphics version 5, reference manual, STSC, Inc 1991

Armand G, Laden P, Rosenberg P (1988) Comment planter et entretenir les résineux (fiche de vulgarisation FVFE et CRPF LorraineAlsace), 4

Birot $Y$, Lanares $R$ (1980) Expérience de provenance de Douglas 1702 (document interne INRA), 32

Causton DR, Venus JC (1981) Single leaf growth and the Richards function. In: The biometry of plant growth. Edward Arnold, Sevenoaks, Kent, 87-100

Conover WJ (1980) Practical nonparametric statistics. John Wiley \& sons, NY, 229-237

Decourt N, Vannière B (1984) Tables de production pour les forêts françaises. ENGREF, Nancy, $237 p$

Jarret $P(1978)$ Variabilité génétique des caractères de forme (tronc et branchaison) et de quelques caractères auxométriques et phénologiques chez le Douglas. Mémoire ENITEF, INRA Orléans, $77 \mathrm{p}$

Kleinschmit J, Svolba J, Weisgerber H, Dimpflmeier R, Ruetz W, Widmaier T (1987) Results of the IUFRO Douglas Fir provenance experiment in the Federal Republic of Germany at age 14. In: Proceedings of the IUFRO working party on breeding strategy for Douglas-Fir as an introduced species. Vienna, Austria, June 1985, IUFRO, 67-75

Magnussen S, Park YS (1991) Growth-curve differentiation among japanese larch provenances. Can J For Res 21, 504-513

Ottorini JM (1991) Growth and development of individual Douglas-fir in stands for applications to simulate in silviculture. Ann Sci For $48,651-666$

Riou-Nivert $P$ (1989) Douglas, qualités du bois, élagage et sylviculture. Rev For Fr XLI 51989, 387-410

Rosette C (1986) Contribution à l'exploration de la variabilité infraspécifique du Douglas. Mémoire ENITEF, INRA Orléans, 137 p

Sprent $P$ (1989) The Kruskall-Wallis and related tests. In: Applied nonparametric statistical methods. Chapman and Hall, 112-134

Sprinz PT, Talbert SZ, Strub MR (1989) Heightage trends from an Arkansas seed source study. Forest Sci 35, 3, 677-691

Tomassone R, Lesquoy E, Millier C (1983) La régression, nouveaux regard sur une ancienne méthode statistique. INRA, actualités scientifiques et agronomiques 13, Masson, Paris, 122-127 